\title{
Papers
}

\section{Systematic review of long term effects of advice to reduce dietary salt in adults}

\author{
Lee Hooper, Christopher Bartlett, George Davey Smith, Shah Ebrahim
}

\begin{abstract}
Objective To assess the long term effects of advice to restrict dietary sodium in adults with and without hypertension.

Design Systematic review and meta-analysis of randomised controlled trials.

Data sources Cochrane library, Medline, Embase, and bibliographies.

Study selection Unconfounded randomised trials that aimed to reduce sodium intake in healthy adults over at least 6 months. Inclusion decisions, validity and data extraction were duplicated. Random effects meta-analysis, subgrouping, sensitivity analysis, and meta-regression were performed.

Outcomes Mortality, cardiovascular events, blood pressure, urinary sodium excretion, quality of life, and use of antihypertensive drugs.

Results Three trials in normotensive people $(\mathrm{n}=2326)$, five trials in those with untreated

hypertension $(\mathrm{n}=387)$, and three trials in people being treated for hypertension $(\mathrm{n}=801)$ were included, with follow up from six months to seven years. The large high quality (and therefore most informative) studies used intensive behavioural interventions. Deaths and cardiovascular events were inconsistently defined and reported. There were 17 deaths, equally distributed between intervention and control groups. Systolic and diastolic blood pressures were reduced (systolic by 1.1 $\mathrm{mm} \mathrm{Hg}, 95 \%$ confidence interval 1.8 to $0.4 \mathrm{~mm} \mathrm{Hg}$; diastolic by $0.6 \mathrm{~mm} \mathrm{Hg}, 1.5$ to $-0.3 \mathrm{~mm} \mathrm{Hg}$ ) at 13 to 60 months, as was urinary 24 hour sodium excretion (by $35.5 \mathrm{mmol} / 24$ hours, 47.2 to 23.9). Degree of reduction in sodium intake and change in blood pressure were not related.

Conclusions Intensive interventions, unsuited to primary care or population prevention programmes, provide only small reductions in blood pressure and sodium excretion, and effects on deaths and cardiovascular events are unclear. Advice to reduce sodium intake may help people on antihypertensive drugs to stop their medication while maintaining good blood pressure control.
\end{abstract}

\section{Introduction}

Several systematic reviews have reported that restricting sodium intake in people with hypertension reduces their blood pressure. ${ }^{1-5}$ However, most of the trials in these systematic reviews were short term and did not allow for complete adjustment of blood pressure to altered sodium intake or reduced motivation for following dietary restrictions over time. Also, some trials increased sodium intake in one arm and compared this with a reduced sodium intake in the other arm and so did not estimate likely effects of cutting down on sodium in a normal diet. ${ }^{6}$ No review on long term outcomes has been carried out since 1998, although large relevant trials have been published.

The value of lowering blood pressure depends on its effects on cardiovascular events and deaths. The published systematic reviews on the effect of salt restriction on blood pressure and other risk factors have disagreed about the size of blood pressure changes $^{8}$ and the effects on cardiovascular events and deaths. We assessed, in people with and without hypertension, the efficacy of advice to reduce dietary sodium intake over at least six months on mortality, cardiovascular events, blood pressure, urinary sodium excretion, quality of life, and use of antihypertensive medications.

\section{Methods}

A previous large scale search for dietary trials and cardiovascular disease covered the Cochrane library, Medline, Embase, CAB abstracts, CVRCT registry, and SIGLE to May 1998 plus bibliographies of collected papers and reviews. ${ }^{9}$ We carried out a further search, seeking trials on sodium restriction and blood pressure in Medline, Embase, and the Cochrane library (to July 2000). We checked bibliographies of systematic reviews and included trials; the searches were not limited by language.

We included trials in which randomisation was adequate, there was a usual or control diet group, the intervention aimed to reduce sodium intake, the intervention was not multifactorial, the participants were not children, acutely ill, pregnant, or institutionalised, follow up was at least 26 weeks, and data on any of the review outcomes were available.

For this review our primary outcomes were mortality and cardiovascular events, blood pressure, and urinary sodium excretion. We also collected data on quality of life and use of antihypertensive medication.

Two authors (LH and $\mathrm{CB}$ ) assessed inclusion and validity and carried out data extraction independently in duplicate. Any differences were resolved by
MANDEC,
University Dental
Hospital of
Manchester,
Manchester
M15 6FH
Lee Hooper
research fellow in
evidence based care
and systematic review
Department of
Social Medicine,
MRC Health
Services Research
Collaboration,
University of
Bristol, Bristol
BS8 2PR
Christopher Bartlett
research associate in
health services
research
George Davey
Smith
professor of clinical
epidemiology
Shah Ebrahim
professor in
epidemiology of
ageing
Correspondence to:
L Hooper
lee.hooper@
man.ac.uk
bmj.com 2002;325:628 
discussion and, when necessary, by a third reviewer (SE). For assessment of quality we collected data on randomisation procedure, allocation concealment, blinding of participants, providers of care, outcome assessors, and losses to follow up. ${ }^{10}$

For urinary sodium excretion and blood pressure we collected data on mean (SD) change from baseline for intervention and control groups at intermediate (latest data point from 6 to 12 months), late (13 to 60 months), and very late (after 60 months) follow up. Four trials provided baseline and follow up values, with SD or SE, but no SD for the change from baseline. ${ }^{11-14}$ We used three studies in which data were provided at baseline and follow up and mean differences given ${ }^{15-17}$ to calculate values for the correlations between baseline and follow up (for the control and experimental groups for systolic and diastolic blood pressure values but not for urinary sodium excretion).$^{18}$ We used a conservative estimate (lowest correlation) to compute the SD of mean changes for four studies without this data. Correlations varied from -5.79 to 0.56 .

In factorial trials of calorie and sodium reduction we used only data from the sodium reduction and control groups because, of three such factorial trials, ${ }^{17} 1920$ two showed definite ${ }^{17}$ or probable ${ }^{19}$ interaction effects. In one trial data on urinary sodium excretion were not available for sodium reduction groups alone but event and medication data were available and were used in analyses. ${ }^{20}$ Calorie reduction and calories plus sodium reduction arms were included in a sensitivity analysis.

We attempted to contact authors of all included trials for further information on trial characteristics, quality, and outcomes (including number and type of cardiovascular events, deaths, quality of life assessments, urinary sodium excretion, intake of other nutrients, blood pressure, and weight) as well as information on further published or unpublished trials.

Two trials were cluster randomised. In one small trial 19 general practitioners were randomised to deliver simple advice on low salt diets or no such advice to 77 patients. ${ }^{13}$ Patient numbers in the intervention and control groups were reduced to an effective sample size as described by Hauck, ${ }^{21}$ assuming the intraclass correlation (appropriate for nonfamilial clusters such as randomised practice units) to be $0.5{ }^{22}$ The other cluster randomised trial individually randomised "index" men and women and then included members of their families in the trial. ${ }^{23}$ We used only the "index" participants in our meta-analysis.

We checked the meta-analyses (weighted mean differences, random effects model, on Cochrane Collaboration Review Manager 4.1 software) for heterogeneity by visual inspection and by Cochran's test. We used sensitivity analysis to assess the robustness of the results to exclusion of the data with estimated SDs, or use of the largest correlations to estimate these SDs, exclusion of trials with unknown or inadequate allocation concealment, and addition of weight reduction arms. ${ }^{102}$ We used the STATA metareg command ${ }^{25}$ for random effects meta-regression. ${ }^{26}$ We did not use funnel plots to investigate the presence of publication bias because the number of trials in each group was too small.

We used subgrouping of trials and meta-regression to examine the effects on blood pressure of length of follow up on sodium excretion and blood pressure, ini- tial systolic blood pressure, presence or absence of hypertension, age, and change in sodium excretion.

\section{Results}

\section{Study characteristics}

Figure 1 shows details of exclusion and inclusion of studies. Table 1 shows the characteristics of the 11 trials included.

We included three trials in people without hypertension ( $\mathrm{n}=2326),{ }^{16}{ }^{17} 19$ five in people with untreated hypertension (n=387), ${ }^{11} 13152327$ and three in people with treated hypertension $(\mathrm{n}=801),{ }^{12}{ }^{140}$ with follow up from six months to seven years. The people without hypertension were healthy (predominantly white men, mean age 40 years) with high normal blood pressure. The people with untreated hypertension were aged 16 to 64 years, while those with treated hypertension were aged 55 to 67 years. In trials on people with hypertension, sex and ethnic characteristics were generally poorly documented. All the trials in people without hypertension, but only one trial in people with treated hypertension, ${ }^{20}$ used a comprehensive behavioural change programme, whereas the others used varying types of advice or leaflets.

The quality of the trials, as judged by concealment of allocation, seemed higher in the trials in people without hypertension. Other aspects of quality that we assessed included blinding of outcome assessment and losses to follow up (table 1). There were different methods of dealing with missing data associated with losses to follow up. Most trials attempted to blind outcome assessors.

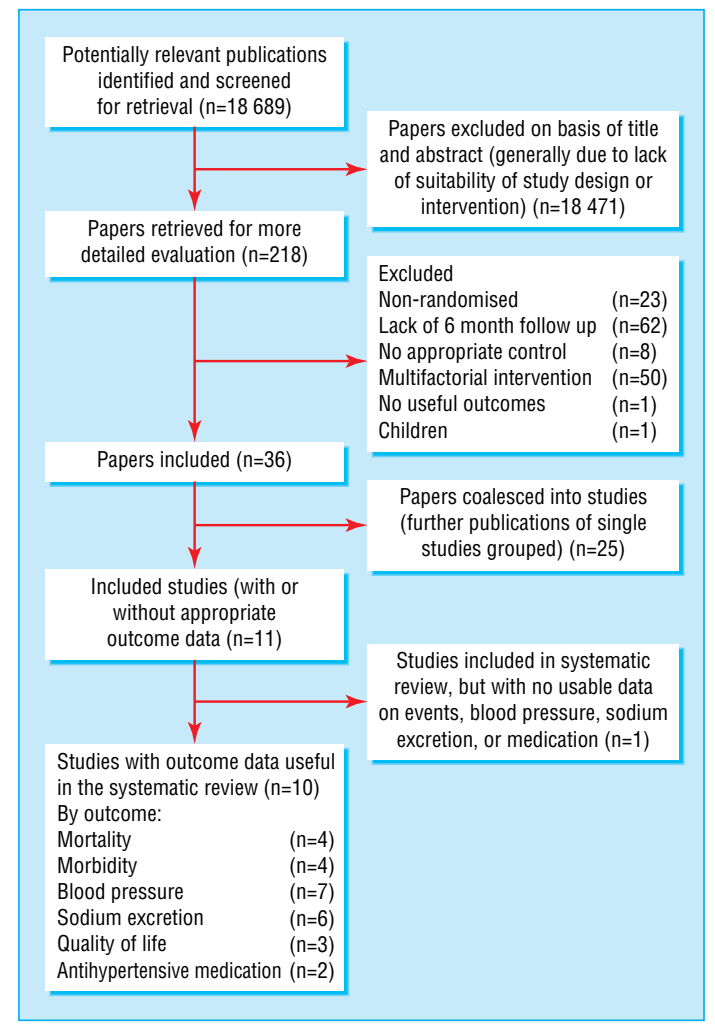

Fig 1 Flow diagram of systematic review (QUOROM statement flow diagram) 
Table 1 Characteristics of trials of sodium restriction included in meta-analysis

\begin{tabular}{|c|c|c|c|c|c|}
\hline Trial name & Participants & Intervention & Control & Outcomes & Quality \\
\hline \multicolumn{6}{|c|}{ People without hypertension } \\
\hline HPT $1990(\text { USA) })^{19}$ & $\begin{array}{l}\text { Mean age } 39 \text { years, } 62 \% \\
\text { men, } 84 \% \text { white, DBP } 78-89 \\
\mathrm{~mm} \mathrm{Hg} \text {, not on AHTM }\end{array}$ & $\begin{array}{l}196 \text { assigned to dietary and } \\
\text { behavioural change programme; } \\
174 \text { followed up at } 6 \text { months, } \\
175 \text { at } 36 \text { months; target USE } \\
\leqslant 70\end{array}$ & $\begin{array}{l}196 \text { assigned to no dietary } \\
\text { counselling; } 191 \text { followed up } \\
\text { at } 6 \text { months, } 178 \text { at } 36 \\
\text { months }\end{array}$ & $\begin{array}{l}\text { BP and USE at } 0,6,36 \\
\text { months, } \% \text { on } \\
\text { antihypertensive drugs }\end{array}$ & $\begin{array}{l}\text { Allocation adequately concealed; } \\
\text { participants not blinded; outcome } \\
\text { assessors blinded; participants } \\
\text { with no follow up excluded; } \\
\text { others given reading from last } \\
\text { visit (or treated BP if higher) }\end{array}$ \\
\hline $\begin{array}{l}\text { TOHP phase I } 1992 \\
\text { (USA)16 }\end{array}$ & $\begin{array}{l}\text { Mean age } 43 \text { years, } 71 \% \\
\text { male, } 77 \% \text { white, DBP } 80 \text { to } \\
89 \mathrm{~mm} \mathrm{Hg} \text {, not on AHTM }\end{array}$ & $\begin{array}{l}327 \text { assigned to group nutrition } \\
\text { and behavioural counselling } \\
\text { programme; } 301 \text { followed up at } \\
12 \text { months, } 304 \text { at } 18 \text { months; } \\
\text { target USE } 80\end{array}$ & $\begin{array}{l}417 \text { assigned to no } \\
\text { intervention; } 392 \text { analysed at } \\
12 \text { months, } 395 \text { at } 18 \text { months }\end{array}$ & $\begin{array}{l}\text { BP and USE at } 0,6,12,18 \\
\text { months }\end{array}$ & $\begin{array}{l}\text { Allocation adequately concealed; } \\
\text { participants not blinded; outcome } \\
\text { assessors blinded; participants } \\
\text { with no follow up reading taken } \\
\text { as zero change, others given } \\
\text { reading from last visit }\end{array}$ \\
\hline $\begin{array}{l}\text { TOHP phase II } 1997 \\
\text { (USA) }^{17}\end{array}$ & $\begin{array}{l}\text { Mean age } 44 \text { years, } 67 \% \\
\text { male, } 81 \% \text { white, DBP } 83 \text { to } \\
89 \mathrm{~mm} \mathrm{Hg} \text {, SBP } \leqslant 140 \mathrm{~mm} \\
\mathrm{Hg} \text {, not on AHTM }\end{array}$ & $\begin{array}{l}594 \text { assigned to dietary and } \\
\text { behavioural change programme, } \\
\text { intensive early on, contact } \\
\text { maintained later; } 529 \text { followed up } \\
\text { at } 6 \text { months, } 515 \text { at } 36 \text { months; } \\
\text { target USE } 70\end{array}$ & $\begin{array}{l}596 \text { assigned to no active } \\
\text { intervention; } 538 \text { analysed at } \\
6 \text { months, } 514 \text { at } 36 \text { months }\end{array}$ & $\begin{array}{l}\text { BP and USE at } 0,6,18,36 \\
\text { months ( } 42 \text { or } 48 \text { months } \\
\text { sometimes) }\end{array}$ & $\begin{array}{l}\text { Allocation adequately concealed } \\
\text { participants not blinded } \\
\text { outcome assessors blinded; } \\
\text { participants with no follow up } \\
\text { reading given random value from } \\
\text { range of results, others given } \\
\text { reading from last visit }\end{array}$ \\
\hline \multicolumn{6}{|c|}{ People with untreated hypertension } \\
\hline $\begin{array}{l}\text { Morgan } 1978 \\
\quad(\text { Australia) })^{1528}\end{array}$ & $\begin{array}{l}>50 \text { years, all men, DBP } \\
95-109 \mathrm{~mm} \mathrm{Hg} \text {, no AHTM }\end{array}$ & $\begin{array}{l}34 \text { for BP ( } 35 \text { for mortality) } \\
\text { assigned to instruction to reduce } \\
\text { their dietary sodium chloride } \\
\text { intake; } 26 \text { followed up at } 24 \\
\text { months (all followed for } \\
\text { mortality); target sodium intake } \\
70-100 \text { mmol/24 hours }\end{array}$ & $\begin{array}{l}33 \text { for BP (42 for mortality) } \\
\text { assigned to no dietary } \\
\text { treatment; } 21 \text { followed up at } \\
24 \text { months (all followed for } \\
\text { mortality) }\end{array}$ & $\begin{array}{l}\text { BP and USE at } 0,6,12,18 \text {, } \\
24 \text { months }\end{array}$ & $\begin{array}{l}\text { Concealment of allocation } \\
\text { unclear; participants not blinded; } \\
\text { outcome assessors blinded; } \\
\text { participants with no follow up } \\
\text { excluded, reading at last visit } \\
\text { used for remainder }\end{array}$ \\
\hline Costa 1981 (Italy) ${ }^{11}$ & $\begin{array}{l}\text { Age range } 16-31 \text { years, } \\
\text { "untreated borderline } \\
\text { hypertension" }\end{array}$ & $\begin{array}{l}21 \text { assigned to receive low salt } \\
\text { diet; } 20 \text { followed up; target } 3 \mathrm{~g} \\
\mathrm{NaCl} / \text { day }\end{array}$ & $\begin{array}{l}20 \text { advised on diet with } \\
\text { unrestricted salt; } 21 \text { [sic] } \\
\text { followed up }\end{array}$ & $\begin{array}{l}\text { BP and intralymphocytic } \\
\text { sodium at } 0 \text { and } 12 \text { months }\end{array}$ & $\begin{array}{l}\text { Concealment of allocation } \\
\text { unclear; participants not blinded; } \\
\text { unclear if outcome assessors } \\
\text { blinded; adjustment for losses } \\
\text { not specified }\end{array}$ \\
\hline $\begin{array}{l}\text { Thaler } 1982 \text { (New } \\
\text { Zealand) }\end{array}$ & $\begin{array}{l}\text { Mean age } 41 \text { years, } 48 \% \\
\text { male, index subjects: SBP } \\
137-180 \mathrm{~mm} \mathrm{Hg}, 21 \% \text { on } \\
\text { AHTM (family members also } \\
\text { included) }\end{array}$ & $\begin{array}{l}80 \text { (38 index }+42 \text { family) } \\
\text { assigned to salt restriction } \\
\text { programme for whole family; } 69 \\
\text { followed up at } 8 \text { months; USE } \\
\text { target not stated }\end{array}$ & $\begin{array}{l}84 \text { ( } 39 \text { index }+45 \text { family) } \\
\text { asked to eat usual diet, } 67 \\
\text { followed up at } 8 \text { months }\end{array}$ & USE at 0 and 8 months & $\begin{array}{l}\text { Concealment of allocation } \\
\text { unclear; participants not blinded; } \\
\text { unclear if outcome assessors } \\
\text { blinded; losses excluded }\end{array}$ \\
\hline Alli 1992 (Italy) 13 & $\begin{array}{l}\text { Mean age } 48 \text { years, } 42 \% \\
\text { men, BMl<30, DBP } 90-104 \\
\text { mm Hg, not on AHTM }\end{array}$ & $\begin{array}{l}40 \text { assigned (by GP } \\
\text { randomisation) to receive low } \\
\text { sodium dietary advice; } 26 \\
\text { followed up at } 12 \text { months; USE } \\
\text { target } \leqslant 80\end{array}$ & $\begin{array}{l}37 \text { assigned (by GP } \\
\text { randomisation) to maintain } \\
\text { usual diet; } 30 \text { followed up at } \\
12 \text { months }\end{array}$ & $\begin{array}{l}\text { BP and USE at 1, 3, 6, 9, } 12 \\
\text { months }\end{array}$ & $\begin{array}{l}\text { Allocation inadequately concealed } \\
\text { (cluster randomisation by GP); } \\
\text { participants not blinded; } \\
\text { assessors not blinded; losses } \\
\text { excluded }\end{array}$ \\
\hline \multicolumn{6}{|c|}{ People with treated hypertension } \\
\hline $\begin{array}{l}\text { Morgan } 1987 \\
\text { (Australia) }^{14}\end{array}$ & $\begin{array}{l}\text { Mean age } 61 \text { years, all men, } \\
\text { DBP }<85 \mathrm{~mm} \mathrm{Hg} \text { on AHTM } \\
\text { (>100 uncontrolled) }\end{array}$ & $\begin{array}{l}10 \text { assigned to low sodium diet; } \\
10 \text { followed up at } 9 \text { months; } \\
\text { target sodium intake } 50-75 \\
\text { mmol/24 hours }\end{array}$ & $\begin{array}{l}10 \text { assigned to maintained } \\
\text { normal diet; } 10 \text { followed up at } \\
9 \text { months }\end{array}$ & $\begin{array}{l}\text { Necessity to restart AHTM } \\
\text { after withdrawal, USE at } 0 \text { and } \\
9 \text { months }\end{array}$ & $\begin{array}{l}\text { Concealment of allocation } \\
\text { unclear; participants not blinded; } \\
\text { outcome assessors blinded; last } \\
\text { BP reading before reinstatement } \\
\text { was used; all had at least one } \\
\text { follow up }\end{array}$ \\
\hline $\begin{array}{l}\text { Arroll } 1995 \text { (New } \\
\text { Zealand) })^{12}\end{array}$ & $\begin{array}{l}\text { Mean age } 55 \text { years, } 52 \% \\
\text { men, on AHTM (DBP }>70 \text { to } \\
105 \mathrm{~mm} \mathrm{Hg} \text { or SBP }>155 \text { to } \\
180 \mathrm{~mm} \mathrm{Hg})\end{array}$ & $\begin{array}{l}51 \text { asked to reduce use of high } \\
\text { salt foods, salt added at table } \\
\text { and in cooking; } 44 \text { followed up } \\
\text { at } 6 \text { months; USE targets not } \\
\text { stated }\end{array}$ & $\begin{array}{l}49 \text { assigned to no } \\
\text { intervention; } 43 \text { followed up at } \\
6 \text { months }\end{array}$ & $\begin{array}{l}\text { BP and AHTM levels after } \\
\text { withdrawal of AHTM at } 0 \text { and } \\
6 \text { months, USE at } 6 \text { months }\end{array}$ & $\begin{array}{l}\text { Concealment of allocation } \\
\text { unclear; participants not blinded; } \\
\text { outcome assessors blinded; } \\
\text { losses excluded from BP } \\
\text { measurement, no adjustment } \\
\text { made for those who decreased } \\
\text { or stopped medication }\end{array}$ \\
\hline TONE $1998\left(\right.$ USA) ${ }^{20}$ & $\begin{array}{l}\text { Mean age } 67 \text { years, } 49 \% \\
\text { men, } 76 \% \text { white, on } A H T M \text {, } \\
\text { DBP }<85 \mathrm{~mm} \mathrm{Hg} \text {, SBP }<145 \\
\mathrm{~mm} \mathrm{Hg}\end{array}$ & $\begin{array}{l}340 \text { assigned to group plus } \\
\text { individual nutrition and } \\
\text { behavioural counselling } \\
\text { programme; } 310 \text { followed up at } \\
30 \text { months; USE target }<80\end{array}$ & $\begin{array}{l}341 \text { assigned to no } \\
\text { counselling; } 314 \text { followed up } \\
\text { at } 30 \text { months }\end{array}$ & $\begin{array}{l}\text { Combined BP, use of AHTM, } \\
\text { and cardiovascular events. } \\
\text { USE at } 0,9,18,30 \text { months }\end{array}$ & $\begin{array}{l}\text { Allocation adequately concealed; } \\
\text { participants not blinded; outcome } \\
\text { assessors blinded; used survival } \\
\text { analysis with censoring to project } \\
\text { proportions free of end points }\end{array}$ \\
\hline
\end{tabular}

AHTM=antihypertensive medication, $\mathrm{SBP}=$ systolic blood pressure, $\mathrm{DBP}=$ diastolic blood pressure, USE $=$ urinary sodium excretion, in $\mathrm{mmol} / 24$ hours, GP=general practitioner.

\section{Mortality and cardiovascular events}

Mortality and cardiovascular events were inconsistently reported. No differences in periods of admission to hospital were seen between intervention groups in the hypertension prevention trial (no further data were provided). ${ }^{19}$ Morgan et al reported that three participants in the control group and two participants on low sodium diets were treated for cardiac failure, with four cardiovascular deaths in the low sodium group and two in the control group. ${ }^{152}$ The trial of non-pharmacological interventions in elderly people recorded a wide range of cardiovascular events: 57 in control participants and 44 in those on low sodium diets (relative risk $0.77,95 \%$ confidence interval 0.41 to 1.14). ${ }^{20}{ }^{29}$ However, only nine of these events were due to stroke or myocardial infarction. Overall, the trials 
Study

Change in systolic blood pressure Hypertension prevention trial $1990^{19}$ Morgan $1978^{15}$

Trials of hypertension (TOHP) I $1992^{16}$

Trials of hypertension (TOHP) II $1997^{17}$

Subtotal $(95 \% \mathrm{Cl})$

Test for heterogeneity $\chi^{2}=2.57, d f=3, P=0.46$

Test for overall effect $\mathrm{z}=3.09, \mathrm{P}=0.002$

Change in diastolic blood pressure Hypertension prevention trial $1990^{19}$ Morgan $1978^{15}$

Trials of hypertension (TOHP) I $1992^{16}$

Trials of hypertension (TOHP) II $1997^{17}$

Subtotal $(95 \% \mathrm{Cl})$

Test for heterogeneity $\chi^{2}=6.71, \mathrm{df}=3, \mathrm{P}=0.082$

Test for overall effect $z=1.31, P=0.19$

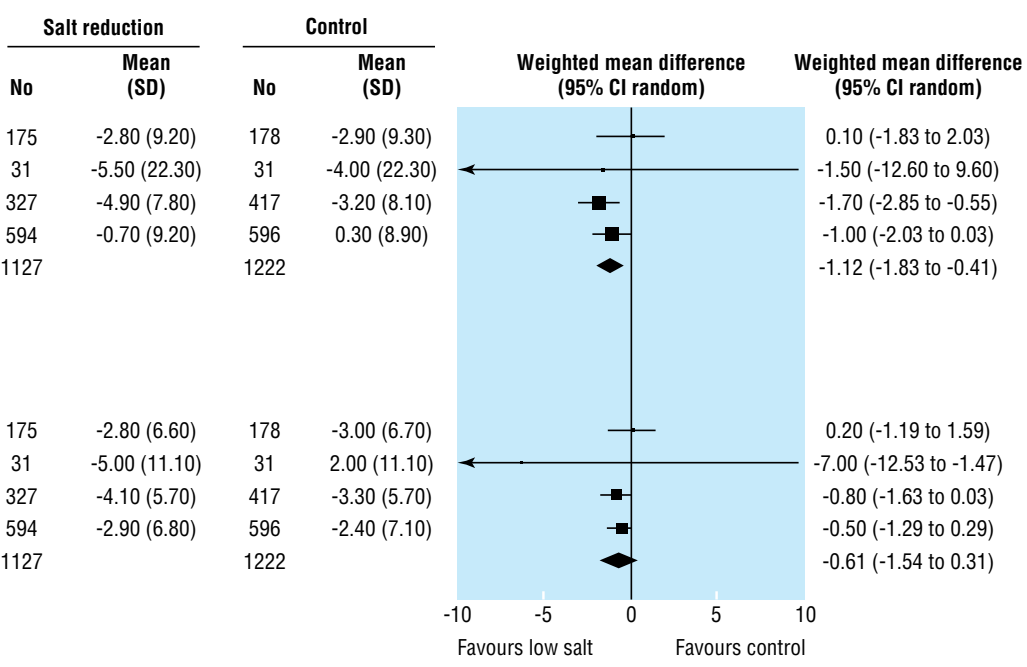

Fig 2 Change in systolic and diastolic blood pressure achieved in trials of 13 to 60 months $(\mathrm{mm} \mathrm{Hg})$

\section{Study}

At 6 to 12 months

Hypertension prevention trial $1990^{19}$

Sliman $1983^{27}$

Trials of hypertension (TOHP) I $1992^{16}$

Trials of hypertension (TOHP) II $1997^{17}$

TONE $1996^{20}$

Thaler (men) $1982^{23}$

Thaler (women) $1982^{23}$

Subtotal $(95 \% \mathrm{Cl})$

At 13 to 60 months

Hypertension prevention trial $1990^{19}$

Trials of hypertension (TOHP) I $1992^{16}$

Trials of hypertension (TOHP) II $1997^{17}$

TONE $1996^{20}$

Subtotal $(95 \% \mathrm{Cl})$

Test for heterogeneity $\chi^{2}=8.31, \mathrm{df}=3, \mathrm{P}=0.04$

Test for overall effect $z=5.96, P=0.00001$

Over 60 months

Trials of hypertension (TOHP) I $1992^{16}$

Subtotal $(95 \% \mathrm{Cl})$

Test for heterogeneity $\chi^{2}=0, \mathrm{df}=0$

Test for overall effect $z=0.85, P=0.4$

\begin{tabular}{ccccc}
\multicolumn{2}{c}{ Salt reduction } & & \multicolumn{2}{c}{ Control } \\
\cline { 1 - 2 } \cline { 5 - 5 } No & $\begin{array}{c}\text { Mean } \\
\text { (SD) }\end{array}$ & & No & $\begin{array}{c}\text { Mean } \\
\text { (SD) }\end{array}$ \\
165 & $-35.70(63.50)$ & & 185 & $-14.80(67.20)$ \\
7 & $-26.40(30.20)$ & & 11 & $26.40(39.80)$ \\
228 & $-55.70(76.10)$ & & 323 & $2.80(80.30)$ \\
99 & $-78.00(86.20)$ & & 101 & $-27.60(108.00$ \\
487 & $-45.20(132.00)$ & & 488 & $1.40(132.00)$ \\
19 & $-64.90(97.90)$ & & 17 & $49.30(67.70)$ \\
18 & $-31.60(55.10)$ & & 18 & $8.40(63.00)$ \\
1023 & & & 1143 &
\end{tabular}

Fig 3 Change in urinary sodium achieved in trials of 6 to 12 months, 13 to 60 months, and >60 months (mmol Na/24 hours) reported few deaths: nine in control groups and eight in low sodium groups.

\section{Blood pressure}

Table 2 shows changes in blood pressure and urinary sodium excretion for each trial, and table 3 shows pooled changes at intermediate and late assessments (fig 2). Reductions in systolic and diastolic blood pressure were apparent at both intermediate $(2.5 \mathrm{~mm} \mathrm{Hg}$, 3.8 to $1.2 ; 1.2 \mathrm{~mm} \mathrm{Hg}, 1.8$ to 0.7 , respectively) and late follow up (1.1 mm Hg, 1.8 to $0.4 ; 0.6 \mathrm{~mm} \mathrm{Hg}, 1.5$ to $-0.3)$. When we carried out sensitivity analyses excluding low quality trials, which included all trials on people with untreated hypertension, the statistical heterogeneity that had been apparent for systolic blood pressure at intermediate follow up and diastolic blood pressure at late follow up was no longer apparent. As these trials were small the effect on pooled estimates of change in blood pressure was minor. Sensitivity analyses including all arms of the factorial trials, ${ }^{17} 19$ suggest that inclusion of weight reduction arms reduces the effect on blood pressure. Meta-regression of change in blood pressure up to 12 months that used all trials with relevant data showed 
Table 2 Results of trials included in meta-analysis. Figures are mean (SD) for blood pressure ( $\mathrm{mm} \mathrm{Hg}$ ) and urinary sodium excreted in $24 \mathrm{hours}$ (mmol) for control and interventions groups

\begin{tabular}{|c|c|c|c|c|c|c|c|c|c|}
\hline Trial name & $\begin{array}{l}\text { Initial } \\
\text { mean } \\
\text { systolic } \\
\text { BP }\end{array}$ & $\begin{array}{l}\text { Change in } \\
\text { systolic BP at } \\
\text { latest point to } 12 \\
\text { months }\end{array}$ & $\begin{array}{l}\text { Change in systolic BP } \\
\text { at latest point after } \\
12 \text { months }\end{array}$ & $\begin{array}{c}\text { Initial } \\
\text { mean } \\
\text { diastolic } \\
\text { BP }\end{array}$ & $\begin{array}{l}\text { Change in diastolic } \\
\text { BP at latest point } \\
\text { to } 12 \text { months }\end{array}$ & $\begin{array}{l}\text { Change in diastolic } \\
\text { BP at latest point } \\
\text { after } 12 \text { months }\end{array}$ & $\begin{array}{l}\text { Initial } \\
\text { urinary } \\
\text { sodium }\end{array}$ & $\begin{array}{l}\text { Change in urinary } \\
\text { sodium at latest } \\
\text { point to } 12 \text { months }\end{array}$ & $\begin{array}{l}\text { Change in urinary } \\
\text { sodium at latest point } \\
\text { after } 12 \text { months }\end{array}$ \\
\hline \multicolumn{10}{|c|}{ People without hypertension } \\
\hline \multicolumn{10}{|c|}{ HPT 1990} \\
\hline Control & 123.9 & $\begin{array}{c}-2.1(8.3)^{*} \text { at } 6 \\
\text { months }\end{array}$ & $\begin{array}{c}-2.9(9.3)^{*} \text { at } 36 \\
\text { months }\end{array}$ & 83.0 & $\begin{array}{c}-3.0(6.9)^{\star} \text { at } 6 \\
\text { months }\end{array}$ & $\begin{array}{c}-3.0(6.7)^{\star} \text { at } 36 \\
\text { months }\end{array}$ & $164.9 \dagger$ & $\begin{array}{c}-14.8(67.2) \dagger \text { at } 6 \\
\text { months }\end{array}$ & 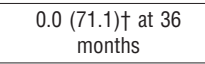 \\
\hline Intervention & 124.0 & $\begin{array}{c}-3.8(7.9)^{\star} \text { at } 6 \\
\text { months }\end{array}$ & $\begin{array}{c}-2.8(9.2)^{*} \text { at } 36 \\
\text { months }\end{array}$ & 82.6 & $\begin{array}{c}-3.4(6.6)^{\star} \text { at } 6 \\
\text { months }\end{array}$ & $\begin{array}{c}-2.8(6.6)^{\star} \text { at } 36 \\
\text { months }\end{array}$ & $162.6 \dagger$ & $\begin{array}{c}-35.7(63.5) \dagger \text { at } 6 \\
\text { months }\end{array}$ & $\begin{array}{c}-16.0(68.0) \dagger \text { at } 36 \\
\text { months }\end{array}$ \\
\hline \multicolumn{10}{|c|}{ TOHP phase I 1992} \\
\hline Control & $\begin{array}{c}125.1 \\
(8.1) \\
\end{array}$ & $\begin{array}{c}-3.9(7.4) \text { at } 12 \\
\text { months }\end{array}$ & $\begin{array}{c}-3.16(8.1) \text { at } 18 \\
\text { months }\end{array}$ & $83.9(2.8)$ & $\begin{array}{c}-3.4(5.7) \text { at } 12 \\
\text { months }\end{array}$ & $\begin{array}{c}-3.3(5.7) \text { at } 18 \\
\text { months }\end{array}$ & $156.4(60.5)$ & $\begin{array}{c}2.8 \text { (80.3) at } 6 \\
\text { months }\end{array}$ & $\begin{array}{c}-11.3(77.7) \text { at } 18 \\
\text { months }\end{array}$ \\
\hline Intervention & $\begin{array}{l}124.8 \\
(8.5)\end{array}$ & $\begin{array}{c}-5.8(7.5) \text { at } 12 \\
\text { months }\end{array}$ & $\begin{array}{c}-4.9(7.8) \text { at } 18 \\
\text { months }\end{array}$ & $83.7(2.7)$ & $\begin{array}{c}-4.4(5.4) \text { at } 12 \\
\text { months }\end{array}$ & $\begin{array}{l}-4.1(5.7) \text { at } 18 \\
\text { months }\end{array}$ & $154.6(59.9)$ & $\begin{array}{c}-55.7(76.1) \text { at } 6 \\
\text { months }\end{array}$ & $\begin{array}{l}-55.2(76.9) \text { at } 18 \\
\text { months }\end{array}$ \\
\hline \multicolumn{10}{|c|}{ TOHP phase II 1997} \\
\hline Control & $\begin{array}{c}127.3 \\
(6.4)\end{array}$ & $\begin{array}{c}-2.2(8.1) \text { at } 6 \\
\text { months }\end{array}$ & $0.3(8.9)$ at 36 months & $85.8(1.9)$ & $\begin{array}{c}-2.8(6.1) \text { at } 6 \\
\text { months }\end{array}$ & $\begin{array}{c}-2.4(7.1) \text { at } 36 \\
\text { months }\end{array}$ & $188.0(80.9)$ & $\begin{array}{c}-27.6(108.0) \text { at } 6 \\
\text { months }\end{array}$ & $\begin{array}{c}-10.5(88.5) \text { at } 36 \\
\text { months }\end{array}$ \\
\hline \multicolumn{10}{|c|}{ People with untreated hypertension } \\
\hline \multicolumn{10}{|c|}{ Morgan 1978} \\
\hline Control & $\begin{array}{c}165 \\
(16.7)\end{array}$ & $\begin{array}{l}-3(22.3) \text { at } 12 \\
\text { months }\end{array}$ & $\begin{array}{l}-4 \text { (22.3) at } 24 \\
\text { months }\end{array}$ & $97(8.6)$ & $1(11.1)$ & $\begin{array}{c}2 \text { (11.1) at } 24 \\
\text { months }\end{array}$ & $191(35)$ & Not given & -11 at 24 months \\
\hline Intervention & $\begin{array}{c}160 \\
(22.3)\end{array}$ & $\begin{array}{c}-3(22.3) \text { at } 12 \\
\text { months }\end{array}$ & $\begin{array}{c}-5.5(22.3) \text { at } 24 \\
\text { months }\end{array}$ & $97(8.7)$ & $-3(11.1)$ & $\begin{array}{c}-5 \text { (11.1) at } 24 \\
\text { months }\end{array}$ & $195(55.0)$ & & -38 at 24 months \\
\hline \multicolumn{10}{|l|}{ Costa 1981} \\
\hline Control & $\begin{array}{c}143.4 \\
(13) \\
\end{array}$ & $\begin{array}{c}4.3(18.8) \neq \text { at } 12 \\
\text { months }\end{array}$ & & $84.1(7)$ & $\begin{array}{c}-0.2(32.6) \ddagger \text { at } 12 \\
\text { months }\end{array}$ & & Not given & & Not given \\
\hline Intervention & $\begin{array}{c}143.3 \\
(15) \\
\end{array}$ & $\begin{array}{l}-14.0(18.5) \ddagger \text { at } \\
12 \text { months }\end{array}$ & & $84.2(9)$ & $\begin{array}{c}-6.1(31.7) \ddagger \text { at } 12 \\
\text { months }\end{array}$ & & & & \\
\hline \multicolumn{10}{|c|}{ Thaler 1982, index men } \\
\hline Control & $139(12)$ & $\begin{array}{c}3.4(17.4) \S \text { at } 12 \\
\text { months }\end{array}$ & & $90(12)$ & $\begin{array}{c}0.8(9.2) \S \text { at } 12 \\
\text { months }\end{array}$ & & $159.5(72.5)$ & $\begin{array}{l}49.3(67.7) \text { at } 12 \\
\text { months }\end{array}$ & \\
\hline Intervention & $137(14)$ & $\begin{array}{c}-5.0(8.3) \S \text { at } 12 \\
\text { months }\end{array}$ & & $86(9)$ & $\begin{array}{c}0.6(9.2) \S \text { at } 12 \\
\text { months }\end{array}$ & & $178.1(76.5)$ & $\begin{array}{c}-64.9(97.9) \text { at } 12 \\
\text { months }\end{array}$ & \\
\hline Intervention & $145(18)$ & $\begin{array}{l}-11.1(24.2) \S \text { at } \\
12 \text { months }\end{array}$ & & $86(11)$ & $\begin{array}{c}-6.8(11.9) \S \text { at } 12 \\
\text { months }\end{array}$ & & $118.0(39.9)$ & $\begin{array}{c}-31.6(55.1) \text { at } 12 \\
\text { months }\end{array}$ & \\
\hline \multicolumn{10}{|l|}{ Silman 1983} \\
\hline Control & 160.5 & $\begin{array}{l}-20.0(24.0) \text { at } \\
12 \text { months }\end{array}$ & & 98.3 & $\begin{array}{c}-11.4(10.5) \text { at } 12 \\
\text { months }\end{array}$ & & 146.5 & $\begin{array}{c}26.4 \text { (39.8) at } 12 \\
\text { months }\end{array}$ & \\
\hline Intervention & 165.3 & $\begin{array}{l}-28.7(26.6) \text { at } \\
12 \text { months }\end{array}$ & & 98.8 & $\begin{array}{c}-17.7(11.4) \text { at } 12 \\
\text { months }\end{array}$ & & 150.8 & $\begin{array}{c}-26.4(30.2) \text { at } 12 \\
\text { months }\end{array}$ & \\
\hline \multicolumn{10}{|l|}{ Alli 1992} \\
\hline Control & $\begin{array}{l}148.3 \\
(10.6)\end{array}$ & $\begin{array}{l}-0.3(16.4) \ddagger \text { at } \\
12 \text { months }\end{array}$ & & $97.2(3.8)$ & $\begin{array}{l}-2.7(16.6) \ddagger \text { at } 12 \\
\text { months }\end{array}$ & & $177.3(61.7)$ & -4.29 at 12 months & \\
\hline Intervention & $\begin{array}{c}150.8 \\
(8.7) \\
\end{array}$ & $\begin{array}{c}-6.6(13.6) \ddagger \text { at } \\
12 \text { months } \\
\end{array}$ & & $97.0(3.1)$ & $\begin{array}{c}-6.4(18.5) \ddagger \text { at } 12 \\
\text { months }\end{array}$ & & $177.3(61.0)$ & 8.6 at 12 months & \\
\hline \multicolumn{10}{|c|}{ People with treated hypertension } \\
\hline \multicolumn{10}{|l|}{ Morgan 1987} \\
\hline Control & $\begin{array}{c}143 \\
(15.8) \\
\end{array}$ & $\begin{array}{c}35(25.7) \ddagger \S \text { at } 9 \\
\text { months }\end{array}$ & & $81(6.3)$ & $17(28.7) \ddagger \S$ & & $163(50.6)$ & -8 & \\
\hline Intervention & $\begin{array}{c}143 \\
(15.8)\end{array}$ & $\begin{array}{c}12(21.5) \ddagger \S \text { at } 9 \\
\text { months }\end{array}$ & & $83(6.3)$ & $7(22.2) \neq \S$ & & $168(37.9)$ & -93 & \\
\hline \multicolumn{10}{|l|}{ Arroll 1995} \\
\hline Control & $\begin{array}{l}145.3 \\
(15.7)\end{array}$ & $\begin{array}{l}-6.2(21.0) \neq \S \text { at } \\
6 \text { months }\end{array}$ & & $94.0(9.8)$ & $\begin{array}{c}-4.8(36.1) \ddagger \S \text { at } 6 \\
\text { months }\end{array}$ & & Not given & Not given & \\
\hline Intervention & $\begin{array}{l}145.4 \\
(15.9)\end{array}$ & $\begin{array}{l}-9.1(21.7) \neq \S \text { at } \\
6 \text { months }\end{array}$ & & $86.4(9.9)$ & $\begin{array}{c}-1.7(34.9) \ddagger \S \text { at } 6 \\
\text { months }\end{array}$ & & & & \\
\hline \multicolumn{10}{|l|}{ TONE 1998} \\
\hline Control & $128(9)$ & & & $71(7)$ & & & 146.2 & $\begin{array}{c}1.4(132)^{\star *} \text { at } 9 \\
\text { months }\end{array}$ & $\begin{array}{c}-0.3(132)^{\star *} \text { at } 30 \\
\text { months }\end{array}$ \\
\hline Intervention & $129(9)$ & & & $72(7)$ & & & 145.3 & $\begin{array}{c}-45.2(132)^{\star \star} \text { at } 9 \\
\text { months }\end{array}$ & $\begin{array}{c}-39.8(143)^{\star *} \text { at } 30 \\
\text { months }\end{array}$ \\
\hline
\end{tabular}

${ }^{*}$ Change data adjusted for baseline differences in composition of treatment groups on 12 covariates

†8 hour overnight urine samples collected, means (SD) adjusted to 24 hour data $(\times 3.8)$.

$¥ S D$ calculated as explained in methods.

$\S$ Levels of antihypertensive medications altered in some participants through study so data not used in meta-analyses.

IData measured off graph, so estimated.

${ }^{* *}$ Data include urinary sodium data for those in weight loss group (added into control, total $n=488$ ) and weight loss plus sodium reduction group (added into intervention, total $n=487$ ) as this information was not available for separate intervention groups. 


\begin{tabular}{|c|c|c|c|}
\hline Type of analysis & $\begin{array}{l}\text { No of } \\
\text { studies }\end{array}$ & $\begin{array}{c}\text { Weighted mean } \\
\text { difference }(95 \% \mathrm{Cl})\end{array}$ & $\begin{array}{l}\mathrm{P} \text { value for } \\
\text { heterogeneity }\end{array}$ \\
\hline \multicolumn{4}{|l|}{ Sodium excretion (mmol Na/24 hours) } \\
\hline Overall analysis (at 6 to 12 months) & 6 & $-48.9(-65.4$ to -32.5$)$ & 0.001 \\
\hline \multicolumn{4}{|l|}{ Sensitivity analysis: } \\
\hline Allocation concealment & 4 & $-43.6(-62.6$ to -24.6$)$ & 0.001 \\
\hline Including weight arms & 6 & $-44.3(-58.4$ to -30.2$)$ & $<0.001$ \\
\hline Overall analysis (at 13 to 60 months) & 4 & $-35.5(-47.2$ to -23.9$)$ & 0.04 \\
\hline \multicolumn{4}{|l|}{ Sensitivity analysis: } \\
\hline Allocation concealment & 4 & $-35.5(-47.2$ to -23.9$)$ & 0.04 \\
\hline Including weight arms & 4 & $-33.3(-42.0$ to -24.6$)$ & 0.05 \\
\hline Overall analysis (at >60 months) & 1 & $10.5(-13.8$ to 34.8$)$ & \\
\hline \multicolumn{4}{|l|}{ Systolic blood pressure (mm Hg) } \\
\hline Overall analysis (at 6 to 12 months) & 7 & $-2.5(-3.8$ to -1.2$)$ & 0.08 \\
\hline \multicolumn{4}{|l|}{ Sensitivity analysis: } \\
\hline Drop calculated SD & 5 & $-2.3(-3.0$ to -1.7$)$ & 0.57 \\
\hline Smallest calculated SD & 7 & $-3.1(-4.8$ to -1.3$)$ & 0.01 \\
\hline Allocation concealment & 3 & $-2.3(-3.1$ to -1.6$)$ & 0.31 \\
\hline Including weight arms & 7 & $-1.6(-3.0$ to -0.2$)$ & $<0.001$ \\
\hline \multicolumn{4}{|l|}{ Subgroups: } \\
\hline No hypertension & 3 & $-2.3(-3.1$ to -1.6$)$ & 0.31 \\
\hline Untreated hypertension & 4 & $-8.0(-15.8$ to -0.2$)$ & 0.15 \\
\hline Overall analysis (at 13 to 60 months) & 4 & $-1.1(-1.8$ to -0.4$)$ & 0.46 \\
\hline \multicolumn{4}{|l|}{ Sensitivity analysis: } \\
\hline Allocation concealment & 3 & $-1.1(-1.9$ to -0.3$)$ & 0.28 \\
\hline Including weight arms & 4 & $-0.5(-1.4$ to 0.4$)$ & 0.10 \\
\hline \multicolumn{4}{|l|}{ Subgroups: } \\
\hline No hypertension & 3 & $-1.1(-1.9$ to -0.3$)$ & 0.28 \\
\hline Untreated hypertension & 1 & $-1.5(-12.6$ to 9.6$)$ & \\
\hline Overall analysis (at >60 months) & 1 & $-3.8(-7.9$ to 0.3$)$ & \\
\hline \multicolumn{4}{|l|}{ Diastolic blood pressure $(\mathrm{mm} \mathrm{Hg})$} \\
\hline Overall analysis (at 6 to 12 months) & 7 & $-1.2(-1.8$ to -0.7$)$ & 0.51 \\
\hline \multicolumn{4}{|l|}{ Sensitivity analysis: } \\
\hline Drop calculated SD & 5 & $-1.2(-1.8$ to -0.6$)$ & 0.31 \\
\hline Smallest calculated SD & 7 & $-1.3(-2.1$ to -0.6$)$ & 0.25 \\
\hline Allocation concealment & 3 & $-1.2(-1.8$ to -0.6$)$ & 0.28 \\
\hline Including weight arms & 7 & $-0.7(-1.5$ to 0.1$)$ & 0.05 \\
\hline \multicolumn{4}{|l|}{ Subgroups: } \\
\hline No hypertension & 3 & $-1.2(-1.8$ to -0.6$)$ & 0.28 \\
\hline Untreated hypertension & 4 & $-4.5(-8.7$ to -0.4$)$ & 0.97 \\
\hline Overall analysis (at 13 to 60 months) & 4 & $-0.6(-1.5$ to 0.3$)$ & 0.08 \\
\hline \multicolumn{4}{|l|}{ Sensitivity analysis: } \\
\hline Allocation concealment & 3 & $-0.5(-1.1$ to 0.0$)$ & 0.48 \\
\hline Including weight arms & 4 & $-0.3(-1.0$ to 0.4$)$ & 0.06 \\
\hline \multicolumn{4}{|l|}{ Subgroups: } \\
\hline No hypertension & 3 & $-0.5(-1.1$ to 0.0$)$ & 0.48 \\
\hline Untreated hypertension & 1 & $-7.0(-12.5$ to -1.5$)$ & \\
\hline Overall analysis (at >60 months) & 1 & $-2.2(-4.8$ to 0.4$)$ & \\
\hline \multicolumn{4}{|l|}{ Dropouts } \\
\hline $\begin{array}{l}\text { Overall analysis in low sodium } v \text { control } \\
\text { groups (latest follow up) }\end{array}$ & 10 & RR=1.04 (0.86 to 1.25$)$ & 0.55 \\
\hline
\end{tabular}

no relation with change in urinary sodium excretion, baseline systolic blood pressure, or age (table 4).

Urinary sodium excretion

We found reductions in urinary 24 hour sodium excretion at both intermediate $(48.9 \mathrm{mmol} / 24$ hours, 65.4 to
32.5) and late follow up (35.5 mmol/24 hours, 47.2 to 23.9) (fig 3). We identified significant heterogeneity in both analyses that was not explained by trial quality. One trial in people without hypertension found that at seven years sodium excretion in a small subset of the original sample was similar in intervention and control groups. $^{30}$

\section{Quality of life}

Information on quality of life was patchy, with no common outcome measures. The hypertension prevention trial asked participants whether they were having problems with their diets. ${ }^{31}$ Of those in the low sodium group, $69 \%$ reported problems such as inconvenience and difficulty with adherence when eating out at some time during the three years of the trial, and problems were reported at $42 \%$ of clinic visits.

The trials of hypertension prevention, phase I (TOHP I), reported psychological wellbeing scores, which improved significantly in participants in the low sodium groups at 18 months compared with the nonintervention control group. ${ }^{32}$ Thaler et al reported that participants did not find it difficult to stop adding salt at table, but many found cutting down on salt in cooking harder. ${ }^{23}$ Most found their low salt bread (salt cut from $2.1 \%$ to $1.0 \%$ dry weight) and salt-free butter acceptable. Only $13 \%$ of participants reported their salt restricted diet as unpleasant or worse. Overall dropout rates, a possible marker of quality of life on trial, were similar (relative risk 1.04; 0.86 to 1.25 ) in low sodium and control groups.

\section{Antihypertensive medications used}

Low salt diets seemed to allow people with hypertension to stop taking medication. In one small trial that compared 10 men in each group, six on low sodium diets had not restarted antihypertensive drugs at six months compared with only one in the control group (relative risk $0.44 ; 0.20$ to 0.98 ). ${ }^{14}$ In a larger study of 975 participants, primary end points (a combination of high blood pressure at any visit, restarting antihypertensive medication, or any clinical cardiovascular disease) were less common in the low sodium group (relative risk $0.83,0.75$ to 0.92 ). ${ }^{20}$

\section{Discussion}

Eleven long term randomised controlled trials of dietary salt reduction (including 3491 participants) provided few data on mortality (17 deaths in total), cardiovascular events, or quality of life but did show significant falls in systolic blood pressure $(1.1 \mathrm{~mm} \mathrm{Hg}$, 1.8 to 0.4 ) and urinary sodium excretion (35.5 mmol/24 hours, 47.2 to 23.9 ) at 13 to 60 months after initial advice. Falls in diastolic blood pressure were smaller and were consistent with no effect $(0.6 \mathrm{~mm} \mathrm{Hg}$, 1.5 to -0.3$)$. A low salt diet may help people on

Table 4 Meta-regression: effects of mean baseline systolic blood pressure, change in sodium excretion, mean age of participants on systolic blood pressure at 6 to 12 months

Explanatory variable Mean baseline systolic blood pressure Mean change in urinary sodium excretion at 6-12 months Slope coefficient $(95 \% \mathrm{Cl})$

\section{Trials in people with and without hypertension} $-0.173(-0.356$ to 0.010$)$ $0.013(-0.049$ to 0.075$)$ $0.118(-0.188$ to 0.424$)$

Constant No of trials

19.5

$-1.68$

$-7.46$

7

Trials in people without hypertension

\begin{tabular}{ccc}
\hline Slope coefficient $(\mathbf{9 5 \%} \mathbf{C l})$ & Constant & No of trials \\
\hline$-0.362(-0.826$ to 0.102$)$ & 43.3 & 3 \\
\hline $0.013(-0.057$ to 0.084$)$ & -1.63 & 3 \\
& & 3 \\
\hline$-0.213(-0.630$ to 0.203$)$ & 6.81 &
\end{tabular}


antihypertensive drugs to stop their medication without loss of blood pressure control.

\section{Limitations and strengths of review}

Health promotion interventions involve several stages before any health outcome is seen. Firstly, the advice must result in changed behaviour (cutting down on salt in food) and, secondly, that behaviour must result in an improved health outcome (reduced cardiovascular illness, increased life expectancy). A major limitation of this review is that we were not able to assess the overall effect of advice to reduce dietary sodium on mortality or morbidity as too few events occurred. Instead we assessed several intermediate outcomes including urinary sodium excretion and blood pressure.

The observed sodium reduction of about a quarter of usual intake in US and UK populations may be an overestimate. $^{33}$ Almost half the participants in one trial ate differently on food record days, eating less food and substituting simpler foods and also eating less salt. ${ }^{34}$ The completeness of urine samples is not known and it has been suggested that less salty foods were eaten on collection days in the trial of Thaler $\mathrm{et}^{\mathrm{a}}{ }^{23}$ (O Simpson, personal communication, 2001).

While both urinary sodium excretion and blood pressure fell, the salt reduction may not have caused the fall in blood pressure. Alterations in diet aimed at reducing salt intake may systematically affect other dietary components (such as alcohol, potassium, or energy intake) that themselves alter blood pressure. This might explain why we found no relation between the degree of reduction in sodium excretion and change in blood pressure. However, the number of

Table 5 Characteristics of systematic reviews on salt and blood pressure

\begin{tabular}{|c|c|c|c|c|c|c|c|c|c|}
\hline \multirow[b]{2}{*}{ Trial } & \multirow{2}{*}{$\begin{array}{c}\text { Inclusion criteria } \\
\text { (population, } \\
\text { intervention, outcome, } \\
\text { design) }\end{array}$} & \multirow{2}{*}{$\begin{array}{l}\text { Only randomised } \\
\text { data included? }\end{array}$} & \multirow{2}{*}{$\begin{array}{c}\text { Normotensive } \\
\text { or } \\
\text { hypertensive }\end{array}$} & \multirow{2}{*}{$\begin{array}{l}\text { Median } \\
\text { (range) } \\
\text { duration of } \\
\text { trials } \\
\end{array}$} & \multirow{2}{*}{$\begin{array}{l}\text { No of trials (No } \\
\text { of participants) }\end{array}$} & \multirow{2}{*}{$\begin{array}{l}\text { Fall in sodium } \\
\text { excretion } \\
\text { (mmol/24 } \\
\text { hours)* }^{*}\end{array}$} & \multicolumn{2}{|c|}{$\begin{array}{l}\text { Pooled mean difference }{ }^{\star}(95 \% \\
\text { CI) }(\mathrm{mm} \mathrm{Hg})\end{array}$} & \multirow{2}{*}{$\begin{array}{l}\text { Quality assessment } \\
\text { and other detail }\end{array}$} \\
\hline & & & & & & & Systolic BP & Diastolic BP & \\
\hline \multirow[t]{2}{*}{$\begin{array}{r}\text { Graudal } \\
1998^{4}\end{array}$} & \multirow{2}{*}{$\begin{array}{l}\text { Mean age >15 years; } \\
\text { low sodium or high } \\
\text { sodium diet, no } \\
\text { confounding; urinary } \\
\text { sodium excretion } \\
\text { measured, systolic, } \\
\text { diastolic or mean BP } \\
\text { reported }\end{array}$} & \multirow[t]{2}{*}{$\begin{array}{l}\text { Yes, random } \\
\text { allocation, } \\
\text { parallel or } \\
\text { crossover }\end{array}$} & Normotensive & $\begin{array}{l}8(4-1100) \\
\text { days }\end{array}$ & $56(2581)$ & 160 & $\begin{array}{c}-1.2 \\
(-0.6 \text { to }-1.8)\end{array}$ & $\begin{array}{c}-0.26 \\
(0.3 \text { to }-0.9)\end{array}$ & \multirow{2}{*}{$\begin{array}{l}\text { Subgrouping by open/ } \\
\text { single blind or double } \\
\text { blind method did not } \\
\text { affect results. } \\
\text { Statistical } \\
\text { heterogeneity noted }\end{array}$} \\
\hline & & & Hypertensive & $\begin{array}{l}28(4-365) \\
\text { days }\end{array}$ & $58(2161)$ & 118 & $\begin{array}{c}-3.9 \\
(-3.0 \text { to }-4.8)\end{array}$ & $\begin{array}{c}-1.9 \\
(-1.3 \text { to }-2.5)\end{array}$ & \\
\hline \multirow[t]{2}{*}{$\begin{array}{l}\text { Midgley } \\
1996^{2}\end{array}$} & \multirow{2}{*}{$\begin{array}{l}\text { Not on antihypertensive } \\
\text { drugs; dietary sodium } \\
\text { intervention; diastolic } \\
\text { and systolic BP } \\
\text { measurement, urinary } \\
\text { sodium excretion; } \\
\text { English language, full } \\
\text { length journal articles }\end{array}$} & \multirow[t]{2}{*}{$\begin{array}{l}\text { Yes, randomised } \\
\text { controlled trials } \\
\text { (crossover or } \\
\text { parallel design) }\end{array}$} & Normotensive & $\begin{array}{l}14 \text { (4-1095) } \\
\text { days }\end{array}$ & $28(2374)$ & $\begin{array}{c}125 \\
\text { (95 to } 156)\end{array}$ & $\begin{array}{c}-1.6 \\
(-2.41 \text { to }-0.89)\end{array}$ & $\begin{array}{c}-0.5 \\
(-1.18 \text { to } \\
0.11)\end{array}$ & \multirow{2}{*}{$\begin{array}{l}\text { Significant } \\
\text { heterogeneity seen, } \\
\text { reduced but not } \\
\text { eliminated when } \\
\text { studies subgrouped } \\
\text { according to quality } \\
\text { characteristics. } \\
\text { Evidence of } \\
\text { publication bias } \\
\text { provided }\end{array}$} \\
\hline & & & Hypertensive & $\begin{array}{l}29(4-730) \\
\text { days }\end{array}$ & $28(1131)$ & $\begin{array}{c}95 \\
\text { (71 to } 119)\end{array}$ & $\begin{array}{c}-5.9 \\
(-7.77 \text { to }-4.12)\end{array}$ & $\begin{array}{c}-3.8 \\
(-4.78 \text { to } \\
-2.9)\end{array}$ & \\
\hline \multirow[t]{2}{*}{ Law $1991^{1} \dagger$} & \multirow{2}{*}{$\begin{array}{l}\text { Not on antihypertensive } \\
\text { drugs; dietary sodium } \\
\text { restriction, not } \\
\text { confounded; } 24 \text { hour } \\
\text { urine collection, systolic } \\
\text { and/or diastolic BP }\end{array}$} & \multirow[t]{2}{*}{ No } & Normotensive & $\begin{array}{l}1.5(0.7 \text { to } 16) \\
\text { weeks }\end{array}$ & $15(?)$ & Not stated & Not stated & Not stated & \multirow{2}{*}{$\begin{array}{l}\text { Quality not assessed. } \\
\text { Individual trial data } \\
\text { compared with pooled } \\
\text { observational data, } \\
\text { rather than pooled } \\
\text { together }\end{array}$} \\
\hline & & & Hypertensive & $\begin{array}{l}5(0.7 \text { to } 104) \\
\text { weeks }\end{array}$ & $63(?)$ & Not stated & Not stated & Not stated & \\
\hline \multirow[t]{2}{*}{ Cutler $1997^{3}$} & \multirow{2}{*}{$\begin{array}{l}\text { Adult; sodium goals } \\
28-273 \text { mmol/24 hours, } \\
\text { no confounding allowed; } \\
\text { lab-based measure of } \\
\text { sodium intake, systolic } \\
\text { and/or diastolic BP } \\
\text { measured }\end{array}$} & \multirow[t]{2}{*}{$\begin{array}{l}\text { Yes, randomised } \\
\text { controlled trials } \\
\text { (crossover or } \\
\text { parallel design), } \\
\text { published only }\end{array}$} & Normotensive & $\begin{array}{l}1 \text { (0.5 to } 36) \\
\text { months }\end{array}$ & 12 (1689) & $\begin{array}{l}\text { Median } \sim 90 \\
\text { (range } 16 \text { to } \\
210 \text { ) }\end{array}$ & $\begin{array}{c}-1.5 \\
(-2.1 \text { to }-1.0)\end{array}$ & $\begin{array}{c}-0.8 \\
(-1.3 \text { to }-0.3)\end{array}$ & \multirow{2}{*}{$\begin{array}{l}\text { Subgrouping by } \\
\text { double blind or not } \\
\text { had no significant } \\
\text { effect on overall } \\
\text { outcome. } \\
\text { Regression analyses } \\
\text { used for publication } \\
\text { bias failed to reject } \\
\text { null hypothesis }\end{array}$} \\
\hline & & & Hypertensive & $\begin{array}{l}2(1-24) \\
\text { months }\end{array}$ & $22(1043)$ & $\begin{array}{l}\text { Median } \sim 71 \\
\text { (range } 27 \text { to } \\
171 \text { ) }\end{array}$ & $\begin{array}{c}-3.8 \\
(-4.9 \text { to }-2.8)\end{array}$ & $\begin{array}{c}-2.1 \\
(-2.8 \text { to }-1.5)\end{array}$ & \\
\hline Alam $1999^{5}$ & $\begin{array}{l}\text { Aged >50 years; } \\
\text { changes in dietary } \mathrm{NaCl} \text {; } \\
\text { blood pressure }\end{array}$ & $\begin{array}{l}\text { Yes, published } \\
\text { English-language } \\
\text { randomised } \\
\text { controlled trials, } \\
\text { crossover or } \\
\text { parallel }\end{array}$ & $\begin{array}{l}\text { Normotensive } \\
(2 \text { trials) or } \\
\text { with essential } \\
\text { hypertension } \\
\text { ( } 9 \text { trials) }\end{array}$ & $\begin{array}{l}14(9-104) \\
\text { weeks }\end{array}$ & $11(485)$ & $\begin{array}{l}\text { Median } 80 \\
\text { (range } 23 \text { to } \\
260 \text { ) }\end{array}$ & $\begin{array}{c}-5.6 \\
(-6.9 \text { to }-4.3)\end{array}$ & $\begin{array}{c}-3.5 \\
(-4.4 \text { to }-2.6)\end{array}$ & $\begin{array}{l}\text { Score tended to be } \\
\text { high (average score } \\
>70 \% \text { ) }\end{array}$ \\
\hline \multirow{2}{*}{$\begin{array}{l}\text { Ebrahim } \\
\text { and } \\
\text { Davey } \\
\text { Smith } \\
1998^{7} \\
\end{array}$} & \multirow{2}{*}{$\begin{array}{l}\text { Adult; dietary sodium } \\
\text { reduction } v \text { control; } \\
\text { diastolic and systolic BP } \\
\text { measurement, urinary } \\
\text { sodium excretion }\end{array}$} & \multirow{2}{*}{$\begin{array}{l}\text { Yes, randomised } \\
\text { controlled trials } \\
\text { of at least } 6 \\
\text { months duration }\end{array}$} & Normotensive & Not stated & 2 (1095) & Not stated & $\begin{array}{c}-1.3 \\
(-2.7 \text { to } 0.1)\end{array}$ & $\begin{array}{c}-0.8 \\
(-1.8 \text { to } 0.2)\end{array}$ & \multirow[t]{2}{*}{ Quality not assessed } \\
\hline & & & Hypertensive & Not stated & $6(466)$ & Not stated & $\begin{array}{c}-2.9 \\
(-5.8 \text { to } 0.0)\end{array}$ & $\begin{array}{c}-2.1 \\
(-4.0 \text { to }-0.1)\end{array}$ & \\
\hline \multirow{2}{*}{$\begin{array}{l}\text { This review, } \\
6-12 \\
\text { months }\end{array}$} & \multirow{4}{*}{$\begin{array}{l}\text { Adult; sodium reduced } \\
\text { diet } v \text { usual diet; urinary } \\
\text { sodium excretion, } \\
\text { systolic and/or diastolic } \\
\text { BP measurements taken } \\
6 \text { to } 12 \text { months or more } \\
\text { than } 13 \text { to } 60 \text { months } \\
\text { after intervention }\end{array}$} & \multirow{4}{*}{$\begin{array}{l}\text { Yes, parallel } \\
\text { randomised } \\
\text { controlled trials }\end{array}$} & Normotensive & $\begin{array}{c}6(6 \text { to } 12) \\
\text { months }\end{array}$ & $3(2326)$ & $\begin{array}{c}43 \\
(16 \text { to } 70) \\
\end{array}$ & $\begin{array}{c}-2.3 \\
(-3.1 \text { to }-1.6) \\
\end{array}$ & $\begin{array}{c}-1.2 \\
(-1.8 \text { to }-0.6) \\
\end{array}$ & \multirow{4}{*}{$\begin{array}{l}\text { Sensitivity analysis } \\
\text { (removing trials } \\
\text { where allocation } \\
\text { concealment is poor } \\
\text { or unclear) had no } \\
\text { effect on direction or } \\
\text { significance of results }\end{array}$} \\
\hline & & & Hypertensive & $\begin{array}{c}12 \text { (12 to } 12) \\
\text { months }\end{array}$ & $4(223)$ & $\begin{array}{c}48 \\
(33 \text { to } 63) \\
\end{array}$ & $\begin{array}{c}-8.0 \\
(-15.7 \text { to }-0.3)\end{array}$ & $\begin{array}{c}-4.3 \\
(-7.1 \text { to }-1.6)\end{array}$ & \\
\hline \multirow{2}{*}{$\begin{array}{c}\text { This review, } \\
13-60 \\
\text { months }\end{array}$} & & & Normotensive & $\begin{array}{l}36(18 \text { to } 36) \\
\text { months }\end{array}$ & $3(2326)$ & $\begin{array}{c}34 \\
(19 \text { to } 50)\end{array}$ & $\begin{array}{c}-1.1 \\
(-1.9 \text { to }-0.3)\end{array}$ & $\begin{array}{c}-0.5 \\
(-1.1 \text { to } 0.0)\end{array}$ & \\
\hline & & & Hypertensive & 24 months & $1(77)$ & $\begin{array}{c}40 \\
\text { (22 to } 57)\end{array}$ & $\begin{array}{c}-1.5 \\
(-12.6 \text { to } 9.6)\end{array}$ & $\begin{array}{c}-7.0 \\
(-12.5 \text { to } \\
-1.5)\end{array}$ & \\
\hline
\end{tabular}

${ }^{*}$ Weighted mean $(95 \% \mathrm{Cl})$ unless stated otherwise. Weighting for all pooled data by inverse variance.

tReview estimates that in people aged 50-59 reduction in $50 \mathrm{mmol} \mathrm{Na} / 24$ hours would lead to reduction of $5 \mathrm{~mm} \mathrm{Hg}$ in systolic and $2.5 \mathrm{mmHg}$ in diastolic blood pressure in people without hypertension and reduction of $7 \mathrm{~mm} \mathrm{Hg}$ and $3.5 \mathrm{~mm} \mathrm{Hg}$, respectively, in people with hypertension. 
trials is small and relating a mean change in blood pressure to a mean change in urinary sodium is statistically weak. In previous meta-analyses an association was seen in some cases but not others (table 5). Data on individual participants are required to take this issue further.

Despite the importance of answering the question of the long term effects of dietary salt restriction, most of the many randomised controlled trials published have been of short duration and can show only that salt restriction is capable of reducing blood pressure but provide no useful information for primary care practice. As randomised controlled trials are available, we have not included population surveys, cohorts, or animal trials that are unable to estimate the unconfounded effects of salt restriction in human populations and are difficult to interpret. ${ }^{35}$

Is it realistic to ask people to alter their salt intake long term? Advice to reduce dietary salt is common in primary care and is a central part of the guidelines produced by the British Hypertension Society. ${ }^{36}$ Despite a great deal of ongoing encouragement and support used in the trials included in this review, it seems that salt reduction attenuates over time. In routine primary care the intervention is likely to be less intense and therefore of more limited impact.

\section{Comparison with previous studies}

It is unclear what effects a low sodium diet has on cardiovascular events and mortality. Lowering sodium intake may have adverse effects on vascular endothelium through stimulation of the renin-angiotensin sys$t^{3} \mathrm{~m}^{37}$ and on serum total and low density lipoprotein cholesterol concentrations. ${ }^{4}$ In cohort studies, lower salt intake in people with hypertension has been associated with higher levels of cardiovascular disease ${ }^{38}$ and in general populations with greater all cause mortality. ${ }^{39}$ However, among obese people lower salt intake may be associated with a reduced risk of cardiovascular events. ${ }^{41}$ These apparently contradictory findings may be explained by confounding or by differential sensitivity to salt intake but make it less clear that salt restriction is without hazards.

We expected that short duration trials would achieve larger reductions in blood pressure that would attenuate over time. As shown in table 5, short term trials of median length of eight days showed a greater reduction in urinary sodium excretion but a similar fall in systolic blood pressure to the findings from long term trials of median length 36 months in this review. The recent dietary approaches to stop hypertension (DASH) trial showed that over a 30 day period with intensive measures, which included provision of all food, systolic blood pressure can fall substantially (by $6.7 \mathrm{~mm} \mathrm{Hg}, 5.4$ to $8.0 \mathrm{~mm} \mathrm{Hg}){ }^{43}$ but this finding is of little relevance to the issue of achieving long term reductions in blood pressure by practical means in primary care.

\section{Implications}

Long term maintenance of low sodium intake is difficult, even with a great deal of support, advice, and encouragement. A policy of reduction in salt intake for the entire population through cutting salt concentrations in processed foods, ${ }^{44}$ as recently announced by the UK chief medical officer, ${ }^{45}$ can achieve small reductions in blood pressure across the whole population for
What is already known on this topic

Restricting sodium intake in people with hypertension reduces blood pressure

Long term effects (on blood pressure, mortality, and morbidity) of reduced salt intake in people with and without hypertension are unclear

\section{What this study adds}

Few deaths and cardiovascular events have been reported in salt reduction trials

Meta-analysis shows that blood pressure was reduced (systolic by $1.1 \mathrm{~mm} \mathrm{Hg}$, diastolic by 0.6 $\mathrm{mm} \mathrm{Hg}$ ) at 13 to 60 months, with a reduction in sodium excretion of almost a quarter (35.5 $\mathrm{mmol} / 24$ hours)

The interventions used were highly intensive and unsuited to primary care or population prevention programmes

Lower salt intake may help people on antihypertensive drugs to stop their medication while maintaining good control of blood pressure, but there are doubts about effects of sodium reduction on overall health

sustained periods of time. Individual reduction of risk would be small, but across a whole population the effects may be substantial. ${ }^{46}$

However, raised blood pressure is only one risk factor for cardiovascular disease and overall clinical benefits (or harms) of a low sodium diet are unclear. Revisiting all participants of the large trials in people without hypertension some years later to assess long term effects of low sodium dietary advice on mortality and cardiovascular morbidity would be a cost effective and relatively rapid way to assess the clinical effectiveness of advice to reduce sodium intake. There is strong justification for a large scale, long term randomised controlled trial to explore the cost effectiveness of such advice if it is to remain a part of the strategy for prevention and treatment of hypertension.

\section{Conclusions}

On present evidence intensive interventions, unsuited to primary care or population prevention programmes, produce uncertain effects on mortality and cardiovascular events and only small reductions in blood pressure. However, advice to reduce sodium intake in the diet may help some people on antihypertensive drugs to stop their medication while maintaining good control of blood pressure.

This study was conducted as a Cochrane systematic review under the auspices of the Cochrane Hypertension and Heart Groups, whose assistance is gratefully acknowledged. We thank all the trialists and experts who kindly provided unpublished information, including Bruce Arroll (University of Auckland), Olaf Simpson (Dunedin), Susan Tonascia (Johns Hopkins University), Trefor Morgan (University of Melbourne), and Alexander Logan (Toronto), as well as Paul Durrington and Helen Worthington ( $\mathrm{PhD}$ supervisors for $\mathrm{LH})$.

Contributors: All authors were actively involved in the design of the review, checking the data, and critical revisions to the manuscript, which was drafted by LH. LH and CB 
independently searched, decided on trial inclusion or exclusion, extracted data, and assessed study quality. LH, CB, and SE performed and duplicated the statistical analyses. SE and GDS were primary advisers, guiding and interpreting the review. $\mathrm{LH}$ is the guarantor.

Funding: North West Research and Development Training Fellowship (LH)

Competing interests: LH owns 285 shares in West Indies Rum Distillery, Barbados.

1 Law MR, Frost CD, Wald NJ. By how much does dietary salt reduction lower blood pressure? III-Analysis of data from trials of salt reduction. BMJ 1991;302:819-24.

2 Midgley JP, Matthew AG, Greenwood CM, Logan AG. Effect of reduced dietary sodium on blood pressure: a meta-analysis of randomized controlled trials. JAMA 1996:275:1590-7.

3 Cutler JA, Follmann D, Allender PS. Randomized trials of sodium reduction: an overview. Am J Clin Nutr 1997;65(suppl 2):643-51S.

4 Graudal NA, Galloe AM, Garred P. Effects of sodium restriction on blood pressure, renin, aldosterone, catecholamines, cholesterols, and triglyceride: a meta-analysis. JAMA 1998;279:1383-91.

5 Alam S, Johnson AG. A meta-analysis of randomised controlled trials (RCT) among healthy normotensive and essential hypertensive elderly patients to determine the effect of high salt $(\mathrm{NaCl})$ diet on blood pressure J Hum Hypertens 1999:13:367-74.

6 Ebrahim S, Davey Smith G. Health promotion in older people for the prevention of coronary heart disease and stroke. London: Health Education Authority, 1996

7 Ebrahim S, Davey Smith G. Lowering blood pressure: a systematic review of sustained effects of non-pharmacological interventions. J Public Health Med 1998;20:441-8.

8 Alderman MH. Population advice on salt restriction: the social issues. Am J Hypertens 2000;13:313-6.

9 Hooper L, Summerbell CD, Higgins JPT, Thompson RL, Clements G, Capps N, et al. Reduced or modified dietary fat for prevention of cardiovascular disease. Cochrane Database Syst Rev 2000;(2):CD002137.

10 Clarke M, Oxman $\mathrm{AD}$, eds. Assessment of study quality. Cochrane reviewer's Handbook 4.1 [updated June 2000]. Section 6. In: The Cochrane Library, Issue 2, 2000 .

11 Costa FV, Ambrosioni E, Montebugnoli L, Paccaloni L, Vasconi L, Magnani B. Effects of low-salt diet and of acute salt loading on blood pressure and intralymphatic sodium concentration in young subjects with borderline hypertension. Clin Sci Lond 1981;61(suppl 7):21-3S

12 Arroll B, Beaglehole R. Salt restriction and physical activity in treated hypertensives. N Z Med J 1995; 108:266-8.

13 Alli C, Avanzini F, Bettelli G, Bonati M, Colombo F, Corso R, et al. Feasibility of a long-term low-sodium diet in mild hypertension. J Hum Hypertens 1999:6:281-6.

14 Morgan T, Anderson A. Sodium restriction can delay the return of hypertension in patients previously well-controlled on drug therapy. Can JPhysiol Pharmacol 1987;65:1752-5.

15 Morgan T, Adam W, Gillies A, Wilson M, Morgan G, Carney S. Hypertension treated by salt restriction. Lancet 1978;i:227-30.

16 Trials of Hypertension Prevention Collaborative Research Group. The effects of nonpharmacologic interventions on blood pressure of person with high normal levels: results of the trials of hypertension prevention, phase I. JAMA 1992;267:1213-20.

17 Trials of Hypertension Prevention Collaborative Research Group. Effects of weight loss and sodium reduction intervention on blood pressure and hypertension incidence in overweight people with high-normal blood pressure. The trials of hypertension prevention, phase II. Arch Intern Med 1997; 157:657-67.

18 Follmann D, Elliott P, Suh I, Cutler JA. Variance imputation for overviews of clinical trials with continuous response. J Clin Epidemiol 1992;45:76973.

19 Hypertension Prevention Trial Research Group. The hypertension prevention trial: three-year effects of dietary changes on blood pressure. Arch Intern Med 1990;150:153-62.

20 Whelton PK, Appel LJ, Espeland MA, Applegate WB, Ettinger WH Jr Kostis JB, et al. Sodium reduction and weight loss in the treatment of hypertension in older persons: a randomized controlled trial of nonpharmacologic interventions in the elderly (TONE). TONE Collaborative Research Group. JAMA 1998;279:839-46.

21 Hauck WW, Gilliss CL, Donner A, gortner S. Randomisation by cluster. Nurs Res 1991;40:356-8.
22 Donner A. An empirical study of cluster randomization. Int J Epidemiol 1982;11:283-6.

23 Thaler BI, Paulin JM, Phelan EL, Simpson FO. A pilot study to test the feasibility of salt restriction in a community. $N$ Z Med J 1982;95:839-42.

24 Schulz KF, Chalmers I, Hayes RJ, Altman DG. Empirical evidence of bias. Dimensions of methodological quality associated with estimates of treatment effects in controlled trials. JAMA 1995;273:408-12.

25 Sharp S. Meta-analysis regression. Stat Tech Bull 1998;42:16-22.

26 Berkley CS, Hoaglin DC, Mosteller F, Colditz GA. A random-effects regression model for meta-analysis. Stat Med 1995;14:395-411.

27 Silman AJ, Locke C, Mitchell P, Humpherson P. Evaluation of the effectiveness of a low sodium diet in the treatment of mild to moderate hypertension. Lancet $1983 ; \mathrm{i}: 1179-82$

28 Morgan TO, Adams WR, Hodgson M, Gibberd RW. Failure of therapy to improve prognosis in elderly males with hypertension. Med J Aust 1980;2:27-31.

29 Kostis JB, Espeland MA, Appel L, Johnson KC, Pierce J, James L. Does withdrawal of antihypertensive medication increase the risk of cardiovascular events? Am J Cardiol 1998;82:1501-8.

$30 \mathrm{He}$ J, Whelton PK, Appel LJ, Charleston J, Klag MJ. Long-term effects of weight loss and dietary sodium reduction on incidence of hypertension. Hypertension 2000;35:544-50.

31 Jeffery RW, French SA, Schmid TL. Attributions for dietary failures: problems reported by participants in the hypertension prevention trial. Health Psychol 1990:9:315-29.

32 Whelton PK, Kumanyika SK, Cook NR, Cutler JA, Borhani NO, Hennekens $\mathrm{CH}$, et al. Efficacy of nonpharmacologic interventions in adults with high-normal blood pressure: results from phase 1 of the trial of hypertension prevention. Trials of hypertension prevention collaborative research group. Am J Clin Nutr 1997;65:652-60S.

33 MAFF. National food survey, 1998. Annual report of food expenditure, consump tion and nutrient intakes. London: HMSO, 1999.

34 Forster JL, Jeffery RW, VanNatta M, Pirie P. Hypertension prevention trial: do 24-h food records capture usual eating behavior in a dietary change study? Am J Clin Nutr 1990;51:253-7.

35 Taubes G. The (political) science of salt. Science 1998:281:898-907.

36 Ramsay LE, Williams B, Johnston G, MacGregor G, Poston L, Potter J, et al. Guidelines for management of hypertension: report of the third working party of the British Hypertension Society. J Hum Hyperten 1999;13:569-92.

37 Alderman MH, Ooi WL, Cohen H, Madhavan S, Sealey JE, Laragh JH. Plasma renin activity: a risk factor for myocardial infarction in hypertensive patients. Am J Hypertens 1997;10:1-8.

38 Alderman MH, Madhavan S, Cohen H, Sealey JE, Laragh JH. Low urinary sodium is associated with greater risk of myocardial infarction among treated hypertensive men. Hypertension 1995;25:1144-52.

39 Alderman MH, Cohen H, Madhavan S. Dietary sodium intake and mortality: the national health and nutrition examination survey (NHANES I). Lancet 1998;351:781-5.

40 Tunstall-Pedoe H, Woodward M, Tavendale R, A'Brook R, McCluskey MK. Comparison of the prediction by 27 different factors of coronary heart disease and death in men and women of the Scottish heart health study: cohort study. BMJ 1997;315:722-9.

41 He J, Ogden LG, Vupputuri S, Bazzano LA, Loria C, Whelton PK. Dietary sodium intake and subsequent risk of cardiovascular disease in overweight adults. JAMA 1999;282:2027-34

42 Tuomilehto J, Jousilahti P, Rastenyte D, Moltchanov V, Tanskanen A, Pietinen P, et al. Urinary sodium excretion and cardiovascular mortality in Finland: a prospective study. Lancet 2001;357:848-51.

43 Sacks FM, Svetkey LP, Vollmer WM, Appel LJ, Bray GA, Harsha D, et al Effects on blood pressure of reduced dietary sodium and the dietary approaches to stop hypertension (DASH) diet. N Engl J Med 2001;344:310

44 MacGregor GA, Sever PS. Salt-overwhelming evidence but still no action: can a consensus be reached with the food industry? BMJ 1996;312:1287-9.

45 Department of Health. The annual report of the chief medical officer of the Department on Health 2001. London, Department of Health, 2001

46 Stamler R. Implications of the INTERSALT study. Hypertension 1991;17(1 suppl):I16-20.

47 Selmer RM, Kristiansen IS, Haglerod A, Graff-Iverson S, Larsen HK, Mever HE, et al. Cost and health consequences of reducing the population intake of salt. J Epidemiol Community Health 2000;54:697-702.

(Accepted 15 May 2002) 